\title{
Is Postmastectomy Radiation Therapy Indicated in Patients with Close or Positive Margins?
}

\author{
Michelle L. Bryan1, Ralph B. D’Agostino², Doris R. Brown³, Marissa M. Howard-McNatt1 ${ }^{*}$ \\ ${ }^{1}$ Surgical Oncology Service, Departments of General Surgery, Wake Forest Baptist School of Medicine, \\ Winston-Salem, NC, USA \\ ${ }^{2}$ Department of Biostatistics, Wake Forest Baptist School of Medicine, Winston-Salem, NC, USA \\ ${ }^{3}$ Department of Radiation Oncology, Wake Forest Baptist School of Medicine, Winston-Salem, NC, USA \\ Email:"mmcnatt@wakehealth.edu
}

Received 6 February 2016; accepted 4 April 2016; published 8 April 2016

Copyright (C) 2016 by authors and Scientific Research Publishing Inc.

This work is licensed under the Creative Commons Attribution International License (CC BY).

http://creativecommons.org/licenses/by/4.0/

\section{(c) (i) Open Access}

\section{Abstract}

Background: The indications for Post Mastectomy Radiation Therapy (PMRT) for positive or close margins are unclear. We examined the indications for PMRT in mastectomy patients with close or positive margins and determined patterns for relapse and survival. Methods: The pathology reports of 610 patients treated with a mastectomy from 1999-2012 were reviewed. Of these, 72 patients had a positive or $<2 \mathrm{~mm}$ margin. Demographic, tumor characteristics, treatments and survival were compared between women treated with and without PMRT. Results: The mean follow up was 4.1 years. Patients who received PMRT were younger $(p=0.03)$ and more likely to receive chemotherapy $(p=0.03)$. Patients with lymphovascular invasion (LVI) were more likely to undergo PMRT $(p=0.02)$. Seven patients who did not receive PMRT recurred. There was no correlation with locoregional failure. The disease free survival was better in the PMRT group $(p=0.03)$, but the overall survival was the same. Conclusion: We found that women with a close or positive margin who were younger, had LVI, and who received chemotherapy received PMRT. The disease free survival was better in the PMRT cohort, but the overall survival was similar. Long-term follow up of patients is warranted to see if PMRT offers a survival advantage.

\section{Keywords}

Postmastectomy Radiation Therapy

\footnotetext{
${ }^{*}$ Corresponding author.
}

How to cite this paper: Bryan, M.L., D’Agostino, R.B., Brown, D.R. and Howard-McNatt, M.M. (2016) Is Postmastectomy Radiation Therapy Indicated in Patients with Close or Positive Margins? Advances in Breast Cancer Research, 5, 66-73. 


\section{Introduction}

Randomized controlled trials have established that Post Mastectomy Radio Therapy (PMRT) improves loco-regional control in patients with invasive breast cancer [1] [2]. Based on these studies, patients at the highest risk for loco-regional recurrence are referred for radiotherapy. Under current guidelines, patients with T3 or greater primary tumor size and/or greater than 4 positive axillary lymph nodes should receive PMRT [3]-[5]. In those patients with early breast cancer (T1-T2) and limited nodal metastasis (1 to 3 positive lymph nodes), adjuvant radiotherapy has been seen as a beneficial method in several studies, although this remains controversial [1] [6]-[8]. To date, there remains a question as to whether patients with a positive or close margin might benefit from PMRT.

Recent studies have addressed some components of this question. Hastings et al. identified a close or positive margin $(\leq 3 \mathrm{~mm})$ as a risk factor for locoregional recurrence in patients with T1 N0 breast cancer [8]. Their study specifically looked at patients who did not receive PMRT, therefore while they recommended that this group may benefit, this was not a direct comparison. Rashtian and colleagues investigated this same question in patients with DCIS and found a margin of $<2 \mathrm{~mm}$ was associated with increased recurrence [9]. Again, this study did not directly compare patients receiving PMRT.

In the present study, our goal was to directly compare patients with close or positive margins who underwent PMRT versus those who did not. We sought to determine any differences in survival and recurrence between the two cohorts.

\section{Materials and Methods}

After approval from the Institutional Review Board, we performed a retrospective cohort study by querying a prospectively maintained database of breast cancer patients for patients who received a mastectomy. From 1999 to 2012 six hundred and ten patients underwent a mastectomy at our institution. Review of the pathology reports yielded 72 patients with a positive or $<2 \mathrm{~mm}$ margin who met the inclusion criteria. Either DCIS or invasive carcinoma at the inked margin was considered a positive margin. DCIS or invasive carcinoma within $<2$ mm was considered a close margin. Patients with an established indication for PMRT, including T3-4 disease or 4 or more lymph nodes with disease were excluded. A retrospective review of these charts was undertaken to discern patient and tumor characteristics. Data relevant to our analysis included demographics, tumor characteristics, receptor status, exposure to neoadjuvant and adjuvant therapies, surgical date and procedure, lymph node status, recurrence, and survival. Patients undergoing simple, modified radical, and skin sparing mastectomy procedures were included. The surgeries were performed by four surgeons over the course of the study.

\section{Statistical Methods}

We summarized patient characteristics using means/standard deviation for continuous variables and frequencies/ percent's for categorical variables. Descriptive statistics were calculated overall by post-mastectomy radiation and any relapse. Significant differences in these groups were assessed using 2 sample t-tests for continuous variables and Chi-square tests or Fisher's Exact tests (depending on the events) for categorical variables. The sample size calculation was statistically significant to show the differences between the two groups. Time to death, time to relapse, or censorship was defined from the date of mastectomy to the date of death or last follow-up on record in our system. Survival analysis methods (Kaplan-Meier approach) were used to compare groups for the time to events. Groups were compared using the likelihood ratio tests. All hypothesis tests performed were two sided and evaluated at the 0.05 significance level. All statistical analysis was performed in SAS Version 9.3 (SAS Institute, Cary, NC).

\section{Results}

Patient characteristics of the 72 total patients included are presented in Table 1 . The total of 72 patients included 57 with close margins of $<2 \mathrm{~mm}, 6$ with margin positive for invasive carcinoma, and 9 with margin positive for DCIS. Of the 57 patients with close margins, 39 were at the deep margin, 16 were at the superficial margin, and two were at both. Patients were predominantly peri/postmenopausal (62\%). Mean age at mastectomy was 53 years old. The type mastectomy performed included simple, modified radical, and skin sparing. Node sampling was performed by sentinel lymph node biopsy (60\%). If a positive sentinel lymph node was detected, completion axillary lymph node dissection (11\%) was performed. One patient with a positive sentinel lymph node did 
Table 1. Patient characteristics.

\begin{tabular}{|c|c|c|}
\hline & Number of Patients & Percentile \\
\hline Characteristic & N or Mean (\%) & N or Mean (\%) \\
\hline Age (years) & 6 & 8 \\
\hline$<40$ & 6 & 8 \\
\hline $41-50$ & 30 & 42 \\
\hline$>50$ & 36 & 50 \\
\hline \multicolumn{3}{|l|}{ Menopausal Status } \\
\hline Premenopausal & 27 & 38 \\
\hline Postmenopausal & 36 & 50 \\
\hline Postmenopausal, surgical & 8 & 11 \\
\hline Perimenopausal & 1 & 1 \\
\hline \multicolumn{3}{|l|}{ Race } \\
\hline Caucasian & 61 & 85 \\
\hline African-American & 8 & 11 \\
\hline Asian & 3 & 4 \\
\hline \multicolumn{3}{|l|}{ Type of Mastectomy } \\
\hline Simple Mastectomy & 29 & 40 \\
\hline Modified radical Mastectomy & 8 & 11 \\
\hline Skin-Sparing Mastectomy & 35 & 49 \\
\hline \multicolumn{3}{|l|}{ Node Sampling } \\
\hline Axillary Lymph Node Dissection & 12 & 17 \\
\hline Sentinel Lymph Node Biopsy & 60 & 83 \\
\hline \multicolumn{3}{|l|}{ Reconstruction } \\
\hline Implant & 49 & 68 \\
\hline Autologous Tissue & 10 & 14 \\
\hline None & 13 & 18 \\
\hline
\end{tabular}

not undergo axillary dissection based upon patient preference. Reconstruction choices in this group included implant (68\%), autologous tissue (14\%), or none (18\%). In cases in which an initial mode of reconstruction was chosen, but later revised, the final mode of reconstruction was reported.

Within the 72 total patients, 17 were identified who underwent PMRT, 55 did not receive PMRT (Table 2). Of the 15 patients with a positive margin for invasive cancer or DCIS, only seven underwent radiation treatments. Ten patients with close margins received radiation treatments. All 17 patients who received PMRT had therapy directed to the chest wall, with 12 out of 17 patients also receiving radiotherapy to the regional nodes. Patients received whole breast chest wall radiation to a total dose of 50 Gray, followed by boost to the scar of 1000 cGy at 200 cGy per fraction for a total of 60 Gray. The 12 patients who also underwent regional nodal radiotherapy received a total dose of 50 Gy in 25 fractions.

The tumor characteristics, patient characteristics and therapy history of the two patient groups were then compared. (Table 2) No statistical difference was found in type of cancer $(\mathrm{p}=0.25)$, tumor size $(\mathrm{p}=0.23)$, histologic grade $(p=0.32)$, or estrogen receptor status $(p=0.08)$. Patients who underwent PMRT were found to be younger with a mean age of 48 versus 54 years $(p=0.03)$. The tumors of those who underwent PMRT were more likely to have lymphovascular invasion (LVI $(\mathrm{p}=0.03)$. In addition, those who underwent PMRT were also more likely to undergo chemotherapy $(p=0.04)$, but less likely to receive endocrine therapy $(p=0.02)$. 
Table 2. Comparison of clinical and tumor characteristics of patients undergoing PMRT versus no PMRT.

\begin{tabular}{|c|c|c|c|c|}
\hline & Overall & No PMRT & Received PMRT & \\
\hline Characteristic & N or Mean (\%) & N or Mean (\%) & N or Mean (\%) & p-value \\
\hline Total patients & 72 & 55 & 17 & -- \\
\hline Age at mastectomy & 60 & 54 & 48 & 0.03 \\
\hline \multicolumn{5}{|l|}{ Type of cancer } \\
\hline DCIS & $8(11.1)$ & $8(14.5)$ & $0(0)$ & \multirow{3}{*}{0.25} \\
\hline Ductal & $56(77.8)$ & $41(74.6)$ & $15(88.2)$ & \\
\hline Lobular & $8(11.1)$ & $6(10.9)$ & $2(11.8)$ & \\
\hline \multicolumn{5}{|l|}{ Invasive tumor size } \\
\hline $0-1 \mathrm{~cm}$ & $14(19.4)$ & $12(21.8)$ & $2(11.8)$ & \multirow{3}{*}{0.23} \\
\hline $1-2 \mathrm{~cm}$ & $28(38.9)$ & $20(36.4)$ & $8(47.1)$ & \\
\hline $2-5 \mathrm{~cm}$ & $22(30.1)$ & $15(27.3)$ & $7(41.2)$ & \\
\hline \multicolumn{5}{|l|}{ Histologic Grade } \\
\hline 0 & $9(12.5)$ & $9(16.4)$ & $0(0)$ & \multirow{4}{*}{0.32} \\
\hline 1 & $12(16.7)$ & $8(14.6)$ & $4(23.5)$ & \\
\hline 2 & $31(43.1)$ & $23(41.8)$ & $8(47.1)$ & \\
\hline 3 & $20(27.8)$ & $15(27.3)$ & $5(29.4)$ & \\
\hline \multicolumn{5}{|c|}{ Lymphovascular invasion (LVI) } \\
\hline Absent & $60(83.3)$ & 49 (89.1) & $11(64.7)$ & \multirow[t]{2}{*}{0.03} \\
\hline Present & $12(16.7)$ & $6(10.9)$ & $6(35.3)$ & \\
\hline \multicolumn{5}{|l|}{ Estrogen receptor status } \\
\hline Negative & $21(29.2)$ & $12(23.6)$ & $8(47.1)$ & \multirow[t]{2}{*}{0.08} \\
\hline Positive & $51(70.8)$ & $42(76.4)$ & $9(52.9)$ & \\
\hline \multicolumn{5}{|c|}{ Underwent chemotherapy } \\
\hline No & $57(79.2)$ & $47(85.5)$ & $10(58.9)$ & \multirow[t]{2}{*}{0.04} \\
\hline Yes & $15(20.8)$ & $8(14.55)$ & $7(41.2)$ & \\
\hline \multicolumn{5}{|c|}{ Received endocrine therapy } \\
\hline No & $51(70.8)$ & 35 (63.6) & $16(94.1)$ & \multirow[t]{2}{*}{0.02} \\
\hline Yes & $21(29.2)$ & $20(36.4)$ & $1(5.9)$ & \\
\hline Recurrence & $7(9.7)$ & $7(12.7)$ & $0(0)$ & 0.19 \\
\hline Local & $4(5.6)$ & $4(7.3)$ & $0(0)$ & 0.57 \\
\hline Distal & $3(4.2)$ & $3(5.5)$ & $0(0)$ & 0.99 \\
\hline
\end{tabular}

Mean overall follow-up was 4.1 years, with average age at mastectomy of 53 years. Overall survival of the entire cohort was 49.5 months. Overall survival from date of mastectomy was compared between patients who underwent PMRT and those who did not. In those who did not undergo PMRT, median survival was 11.1 years (mean 9.91 years) versus not reached in those who underwent PMRT ( $p=0.08$ ) (Figure 1 ).

Recurrence was analyzed as a measure of PMRT effect. Overall, there were 7 recurrences in the 72 patients. Of these, 4 were local recurrences and 3 were distant. The four local recurrences include one in the axilla and three in the chest wall. Of these, one had a positive deep margin, one had a deep margin close for invasive carcinoma, and two had a deep margin close for DCIS. All seven recurrences happened in the group that did not receive PMRT. Comparing recurrence free survival of the two groups yielded a median survival which was not 


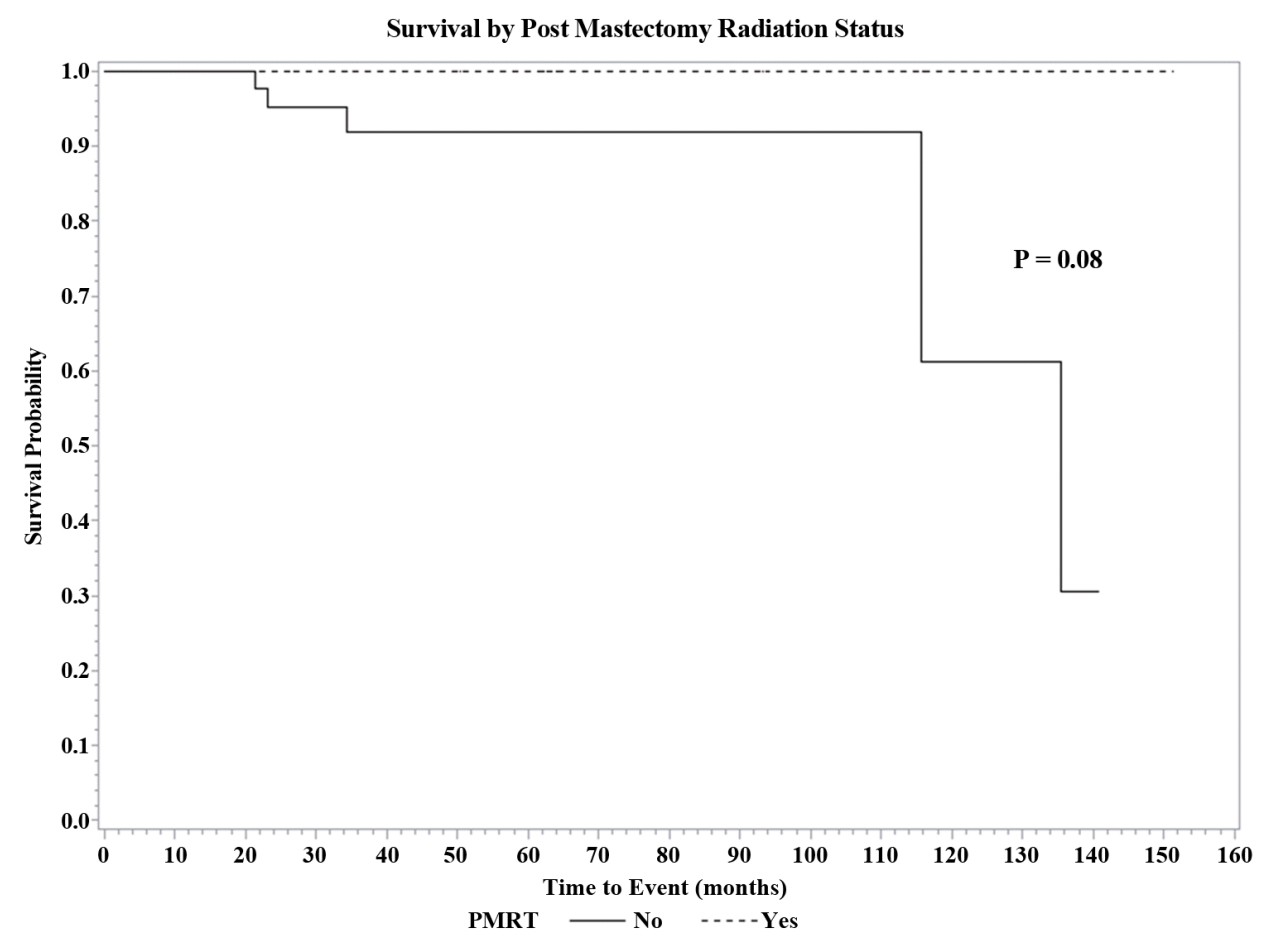

Figure 1. Overall Survival post mastectomy stratified by receipt of PMRT.

reached in both groups (Figure 2). Although, the separation of the two curves did yield a statistical difference (p $=0.03$ ), indicating that with longer follow-up, a measurable median disease free survival may be obtained. A potential bias might have been an increased offering of PMRT to those with positive margins. Of the six patients with positive invasive margin, four received radiation, two did not. Of the nine patients with positive DCIS at the margin, three received radiation, six did not. Together, this indicates that not all patients with a positive margin received PMRT, making this bias less likely.

In order to ascertain whether any pattern could be determined in the patients who recurred, patient characteristics, tumor characteristics, and adjuvant treatment status was analyzed (Table 3). The patients who recurred did not differ from the rest of the subjects in regards to age $(p=0.26)$, type of cancer $(p=0.33)$, histologic grade (0.72), presence of lymphovascular invasion (0.33), estrogen receptor status $(0.41)$, receipt of chemotherapy $(p=$ 0.63 ) or receipt of endocrine therapy $(\mathrm{p}=0.67)$. None of the recurrences happened in the patients with DCIS, yielding a statistically significant $\mathrm{p}$ value of 0.05 . While the number of recurrences was too small to be statistically significant in further subgroups, we did examine the subgroups of close versus positive and margin location in recurrence. There was no direct correlation with margin type, close versus positive. Of the total recurrences, five recurrences were in the close margin group while two were in the positive margin group. In terms of location of margin, five of the recurrences were associated with a positive/close deep margin; two were at the superficial margin.

\section{Discussion}

This study aimed to evaluate the role of PMRT in patients with positive and close margins from the total mastectomy patients of one institution over a 14 year span. The purpose was to extend previously published studies from multiple institutions which have described the risk factors for recurrence. Here, we specifically addressed whether PMRT conveyed a benefit in prevention of recurrence or overall survival.

Recently the EBCTCG (Early Breast Cancer Trialists' Collaborative Group) performed a metaanalysis of 8135 women who received adjuvant radiotherapy versus no radiotherapy after mastectomy for invasive cancer [7]. They found that radiotherapy reduced both recurrence and breast cancer specific mortality in the women with one to three positive lymph nodes, [(overall recurrence (RR 0.68, 95\% CI 0.57-0.82, $2 \mathrm{p}=0.00006$ )], and breast cancer mortality (RR 0.80, 95\% CI 0.67-0.95, $2 \mathrm{p}=0.01$ ) [7]. This adds additional information regarding 
Table 3. Comparison of clinical and tumor characteristics of patients with recurrence versus no recurrence.

\begin{tabular}{|c|c|c|c|}
\hline & No recurrence & Recurrence & \\
\hline Characteristic & N or Mean (\%) & N or Mean (\%) & p-value \\
\hline Total patients & 65 & 7 & -- \\
\hline Age at mastectomy & 52.5 & 57.4 & 0.26 \\
\hline \multicolumn{4}{|l|}{ Type of cancer } \\
\hline DCIS & $8(12.3)$ & $0(0)$ & \\
\hline Ductal & $49(75.4)$ & $7(100)$ & 0.33 \\
\hline Lobular & $8(12.3)$ & $0(0)$ & \\
\hline \multicolumn{4}{|l|}{ Tumor size } \\
\hline $0-1 \mathrm{~cm}$ & $10(15.4)$ & $4(57.1)$ & \\
\hline $1-2 \mathrm{~cm}$ & 27 (41.5) & $1(14.3)$ & 0.05 \\
\hline $2-5 \mathrm{~cm}$ & $20(30.8)$ & $2(28.6)$ & \\
\hline DCIS & $8(12.3)$ & $0(0)$ & \\
\hline \multicolumn{4}{|l|}{ Histologic Grade } \\
\hline 0 & 9 (13.9) & $0(0)$ & \\
\hline 1 & $11(16.9)$ & $1(14.3)$ & 0.72 \\
\hline 2 & $27(41.5)$ & $4(57.1)$ & \\
\hline 3 & $18(27.7)$ & $2(28.6)$ & \\
\hline \multicolumn{4}{|c|}{ Lymphovascular invasion (LVI) } \\
\hline Absent & $55(84.6)$ & $5(71.4)$ & 0.33 \\
\hline Present & $10(15.4)$ & $2(28.6)$ & \\
\hline \multicolumn{4}{|l|}{ Estrogen receptor status } \\
\hline Negative & $18(27.7)$ & $3(42.9)$ & 0.41 \\
\hline Positive & $47(72.3)$ & $4(57.1)$ & \\
\hline \multicolumn{4}{|l|}{ Underwent chemotherapy } \\
\hline No & $52(80.0)$ & $5(71.4)$ & 0.63 \\
\hline Yes & $13(20.0)$ & $2(28.6)$ & \\
\hline \multicolumn{4}{|c|}{ Received endocrine therapy } \\
\hline No & $45(69.2)$ & $6(85.7)$ & 0.67 \\
\hline Yes & $20(30.8)$ & $1(14.3)$ & \\
\hline
\end{tabular}

the benefit of PMRT for women with 1 - 3 positive nodes. However, little data exists for the benefit of PMRT in women with close or positive margins. While the benefits of PMRT in defined populations has been well elucidated in large randomized controlled trials, the benefits for women whom do not fit those risk factors, but have other factors for recurrence, such as close or positive margin, remain controversial.

In 1998, Freedman and colleagues reported on 34 patients with T1-2 N0-1 tumors with margins <1 cm [10]. The cumulative chest wall recurrence was $18 \%$ at 8 years old. The only significant factor for locoregional recurrence was age, with LRR being 28\% for patients younger than 50 years old and $0 \%$ for patients older than 50 years old. Truong et al. analyzed women with positive margins, with 94 patients meeting their inclusion criteria [11]. In their study only some patients received radiotherapy. However, they concluded that not all patients with node-negative breast cancer with positive margins after mastectomy require radiotherapy. Locoregional failure rates approximating $20 \%$ were observed in women with positive margins plus at least one of the following factors: age $\leq 50$ years old, T2 tumor size, grade III histology, or LVI. 


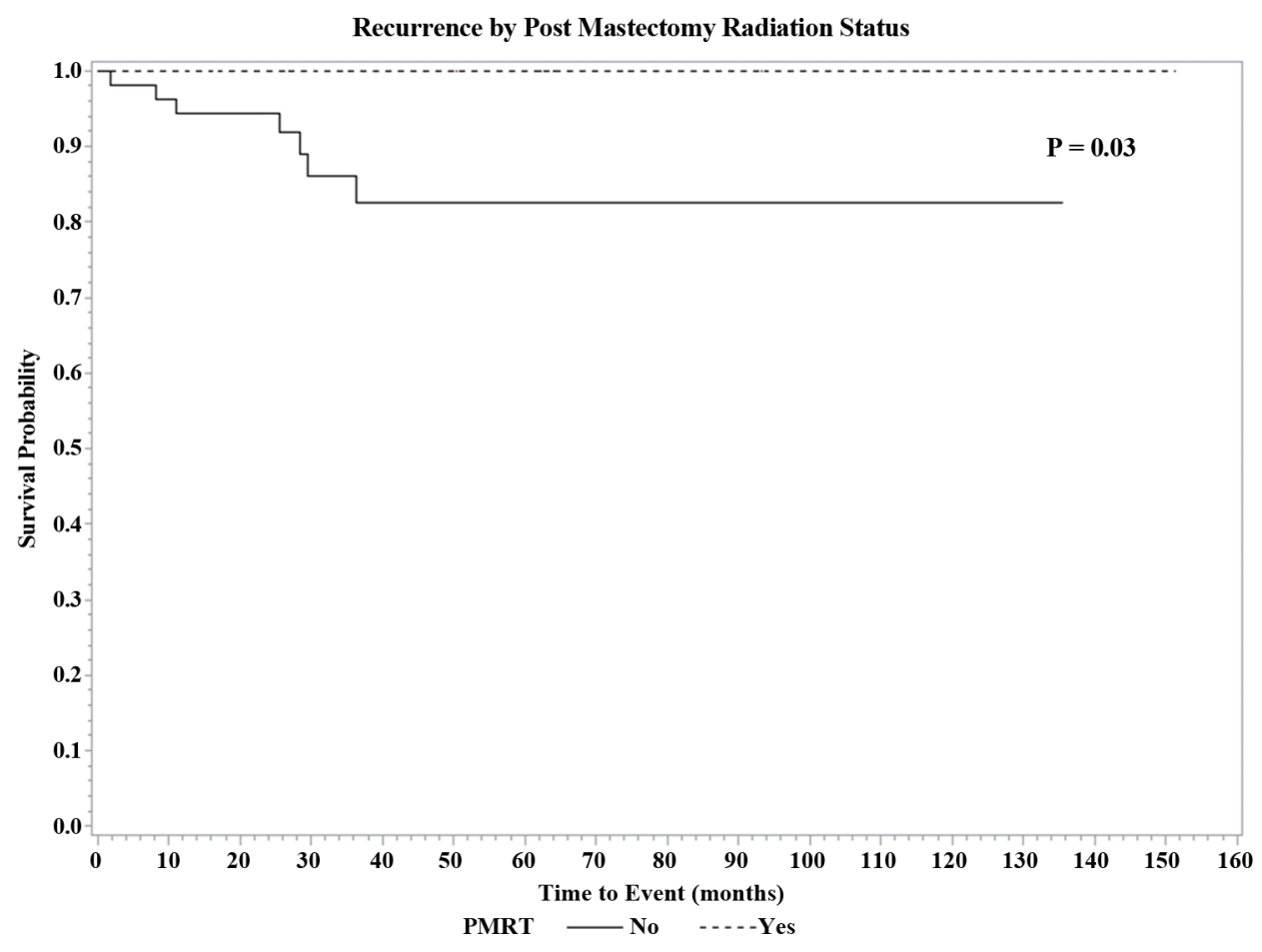

Figure 2. Recurrence post mastectomy stratified by receipt of PMRT.

Hastings et al. analyzed 1259 women with T1 N0 breast cancers treated with mastectomy and no radiation and sought to identify a group at increased risk for local regional recurrence [8]. They found that women with grade 3 tumors $(p=0.002)$ and margins $<3 \mathrm{~mm}(\mathrm{p}=0.02)$ were at an increased risk for local regional recurrence and may benefit from PMRT [8]. MD Anderson examined patients with DCIS treated with mastectomy that had a close or positive margin [12]. Of the 94 patients only seven received PMRT and none had a locoregional recurrence (LRR). Among the remaining 803 patients, the 10 -year LRR rate was 1\% (5.0\% for margins $<1 \mathrm{~mm}$ [p $<0.001]$ ). They concluded that PMRT is warranted only for patients with multiple close or positive margins.

Our study is one of the few that compares women with close or positive margins whom did and did not receive PMRT. Like the above studies, we found that women who were younger and had LVI were more likely to receive PMRT. This likely reflects some selection bias with practitioners suspecting a more aggressive disease type despite there being no difference in the estrogen receptor status between the two groups. Seven patients had disease recurrence, of which four were local recurrences, with all of the recurrences occurring in the non PMRT group. In specifically analyzing this group, we found no correlation with locoregional failure as far as age, ER status, LVI, or grade which contradicts some of the previous mentioned studies. Patients with DCIS did not recur, likely reflecting the less aggressive nature of this disease.

Furthermore, we found that the disease free survival was better in the PMRT group ( $p=0.03)$, although this test was based on analysis with a limited number of events. The majority of women in the PMRT group also received adjuvant chemotherapy. Systemic therapy can approximately halve the 5-year risks of both local and distant recurrence [13]. The addition of chemotherapy likely improved these women's 5-year disease survival. However, the overall survival between the two groups did not reach statistical significance. This may be due to too few events being captured in the time frame.

The main limitation of our study is the small sample size of the number of women with a close margin undergoing PMRT which causes an imbalance between the two groups. Given that PMRT is not currently standard of care in patients with negative ink margin, this was expected in the design. The fact that statistically significant differences do exist between these groups and that trends such as recurrence all fall into this group indicates that this highly selective group requires further study in the future. Given the highly selective nature of this group of patient, such trials would likely need to be multicenter to accrue large numbers. We anticipate that our study will add to the growing body of literature suggesting that investment in larger scale, prospective study of the role of 
PMRT in close margins is indicated.

\section{Conclusion}

We found that women with a close or positive margin who were younger, had LVI, and who received chemotherapy were more likely to receive PMRT. There were no recurrences in the PMRT group. The disease free survival was better in this cohort, but the overall survival was the same between the groups. Further long-term follow up of patients with close or positive margins is warranted to see if PMRT offers a survival advantage.

\section{References}

[1] Overgaard, M., Hansen, P.S., Overgaard, J., Rose, C., Andersson, M., Bach, F., et al. (1997) Postoperative Radiotherapy in High-Risk Premenopausal Women with Breast Cancer who Receive Adjuvant Chemotherapy: Danish Breast Cancer Cooperative Group 82b Trial. The New England Journal of Medicine, 337, 949-955. http://dx.doi.org/10.1056/NEJM199710023371401

[2] Ragaz, J., Jacjson, S.M., Le, N., Plenderleith, I.H., Spinelli, J.J., Basco, V.E., et al. (1997) Adjuvant Radiotherapy and Chemotherapy in Node-Positive Premenopausal Women with Breast Cancer. The New England Journal of Medicine, 337, 956-962. http://dx.doi.org/10.1056/NEJM199710023371402

[3] Recht, A., Edge, S.B., Solin, L.J., Robinson, D.S., Estabrook, A., Fine, R.E., et al. (2001) Postmastectomy Radiotherapy: Clinical Practice Guidelines of the American Society of Clinical Oncology. Journal of Clinical Oncology, 19, 1539-1569.

[4] Taylor, M.E., Haffty, B.G., Rabinovitch, R., Arthur, D.W., Halberg, F.W., Strom, E.A., et al. (2009) ACR Appropriateness Criteria on Postmastectomy Radiotherapy. Expert Panel on Radiation Oncology-Breast. International Journal of Radiation Oncology, Biology, Physics, 73, 997-1002. http://dx.doi.org/10.1016/j.ijrobp.2008.10.080

[5] Truong, P.T., Olivotto, I.A., Whelan, T.J. and Levine, M. (2004) Clinical Practice Guidelines for the Care and Treatment of Breast Cancer: 16. Locoregional Post-Mastectomy Radiotherapy. Canadian Medical Association Journal, 170, 1263-1273. http://dx.doi.org/10.1503/cmaj.1031000

[6] Overgaard, M., Jensen, M.B., Overgaard, J., Hansen, P.S., Rose, C., Andersson, M., et al. (1999) Postoperative Radiotherapy in High-Risk Postmenopausal Breast-Cancer Patients Given Adjuvant Tamoxifen: Danish Breast Cancer Cooperative Group DBCG 82c Randomized Trial. Lancet, 353, 1641-1648. http://dx.doi.org/10.1016/S0140-6736(98)09201-0

[7] EBCTCG Early Breast Cancer Trialists’ Collaborative Group (2014) Effect of Radiotherapy after Mastectomy and Axillary Surgery on 10-Year Recurrence and 20-Year Breast Cancer Mortality: Meta-Analysis of Individual Patient Data for 8135 Women in 22 Randomized Trials. Lancet, 383, 2127-2135. http://dx.doi.org/10.1016/S0140-6736(14)60488-8

[8] Hastings, J., Iganej, S., Huang, C., Huang, R. and Slezak, J. (2014) Risk Factors for Locoregional Recurrence after Mastectomy in Stage T1 N0 Breast Cancer. American Journal of Clinical Oncology, 37, 486-491. http://dx.doi.org/10.1097/COC.0b013e31827e54c2

[9] Rashtian, A., Iganej, S., Amy Liu, I.L. and Natarajan, S. (2008) Close or Positive Margins after Mastectomy for DCIS: Pattern of Relapse and Potential Indications for Radiotherapy. International Journal of Radiation Oncology, Biology, Physics, 72, 1016-1020. http://dx.doi.org/10.1016/j.ijrobp.2008.06.1954

[10] Freedman, G.M., Fowble, B.L., Hanlon, A.L., Myint, M.A., Hoffman, J.P., Sigurdson, E.R., et al. (1998) A Close or Positive Margin after Mastectomy Is Not an Indication for Chest Wall Irradiation Except in Women Aged Fifty or Younger. International Journal of Radiation Oncology, Biology, Physics, 41, 599-605. http://dx.doi.org/10.1016/S0360-3016(98)00103-5

[11] Truong, P.T., Olivotto, I.A., Speers, C.H., Way, E.S., Berthelet, E. and Kader, H.A. (2004) A Positive Margin Is Not Always an Indication for Radiotherapy after Mastectomy in Early Breast Cancer. International Journal of Radiation Oncology, Biology, Physics, 58, 797-804. http://dx.doi.org/10.1016/S0360-3016(03)01626-2

[12] FitzSullivan, E., Lari, S.A., Smith, B., Caudle, A.S., Krishnamurthy, S., Lucci, A., et al. (2013) Incidence and Consequence of Close Margins in Patients with Ductal Carcinoma-In Situ Treated with Mastectomy: Is Further Therapy Warranted? Annals of Surgical Oncology, 20, 4103-4112. http://dx.doi.org/10.1245/s10434-013-3194-0

[13] Early Breast Cancer Trialists’ Collaborative Group (EBCTCG) (2005) Effects of Chemotherapy and Hormonal Therapy for Early Breast Cancer on Recurrence and 15-Year Survival: An Overview of the Randomised Trials. Lancet, 36, 1687-1717. 